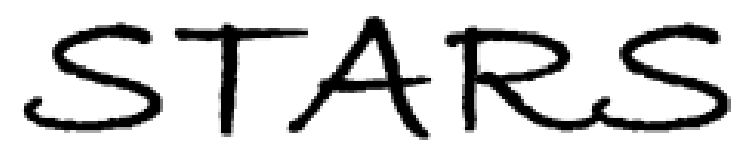

University of Central Florida

STARS

$1-1-2007$

\title{
Sharp estimates on minimum travelling wave speed of reaction diffusion systems modelling autocatalysis
}

Xinfu Chen

Yuanwei Qi

University of Central Florida

Find similar works at: https://stars.library.ucf.edu/facultybib2000

University of Central Florida Libraries http://library.ucf.edu

This Article is brought to you for free and open access by the Faculty Bibliography at STARS. It has been accepted for inclusion in Faculty Bibliography 2000 s by an authorized administrator of STARS. For more information, please contact STARS@ucf.edu.

\section{Recommended Citation}

Chen, Xinfu and Qi, Yuanwei, "Sharp estimates on minimum travelling wave speed of reaction diffusion systems modelling autocatalysis" (2007). Faculty Bibliography 2000s. 6941.

https://stars.library.ucf.edu/facultybib2000/6941

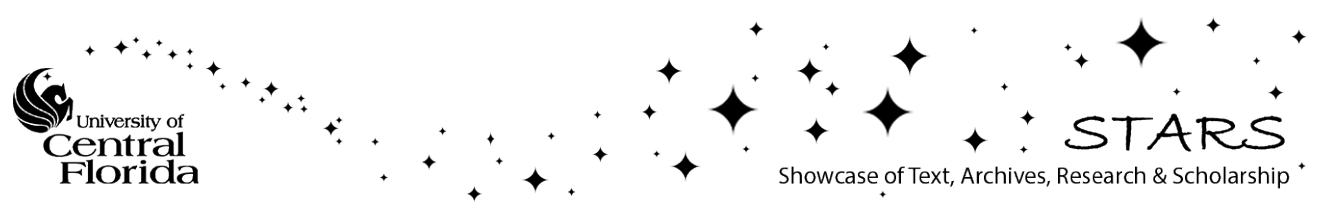




\title{
SHARP ESTIMATES ON MINIMUM TRAVELLING WAVE SPEED OF REACTION DIFFUSION SYSTEMS MODELLING AUTOCATALYSIS*
}

\author{
XINFU CHEN ${ }^{\dagger}$ AND YUANWEI $\mathrm{QI}^{\ddagger}$
}

\begin{abstract}
This article studies propagating wave fronts in an isothermal chemical reaction $A+2 B \rightarrow 3 B$ involving two chemical species, a reactant $A$ and an autocatalyst $B$, whose diffusion coefficients, $D_{A}$ and $D_{B}$, are unequal due to different molecular weights and/or sizes. Explicit bounds $v_{*}$ and $v^{*}$ that depend on $D_{B} / D_{A}$ are derived such that there is a unique travelling wave of every speed $v \geq v^{*}$ and there does not exist any travelling wave of speed $v<v_{*}$. New to the literature, it is shown that $v_{*} \propto v^{*} \propto D_{B} / D_{A}$ when $D_{B} \leq D_{A}$. Furthermore, when $D_{A} \leq D_{B}$, it is shown rigorously that there exists a $v_{\min }$ such that there is a travelling wave of speed $v$ if and only $v \geq v_{\text {min }}$. Estimates on $v_{\text {min }}$ significantly improve those of early works. The framework is built upon general isothermal autocatalytic chemical reactions $A+n B \rightarrow(n+1) B$ of arbitrary order $n \geq 1$.
\end{abstract}

Key words. cubic autocatalysis, travelling wave, minimum speed, reaction diffusion

AMS subject classifications. 34C20, 34C25, 92E20

DOI. $10.1137 / 060665749$

1. Introduction. In this paper we consider an isothermal autocatalytic chemical reaction step governed by the cubic reaction relation

$$
A+2 B \rightarrow 3 B \quad \text { with rate } k a b^{2} .
$$

Here, $k>0$ is the reaction rate, and $a$ and $b$ are the concentrations of reactant $A$ and autocatalyst $B$, respectively. Well documented in the literature, the cubic reaction relation has appeared in several important models of real chemical reactions, e.g., almost isothermal flames in the carbon-sulphide-oxygen reaction (Voronkov and Semenov [22]), iodate-arsenous acid reactions (Saul and Showwalter [19]), hydroxylaminenitrate reactions (Gowland and Stedman [10]), as well as other applications (Aris, Gray, and Scott [1] and Sel'kov [20]).

Experimental observations demonstrate the existence of propagating chemical wave fronts in chemical systems for which cubic catalysis forms a key step $[11,12,13$, 24]. These wave fronts, or travelling waves, arise due to the interaction of reaction and diffusion. Quite often when a quantity of autocatalyst is added locally into an expanse of reactant which is initially at uniform concentration, the ensuing reaction is observed to generate wave fronts which propagate outward from the initial reaction zone, consuming fresh reactant ahead of the wave front as it propagates. This is the phenomenon to be addressed in this paper.

For this purpose, we consider a one-dimensional slab geometry and the following partial differential equations (PDEs) that govern mass concentration and molecular

\footnotetext{
${ }^{*}$ Received by the editors July 21, 2006; accepted for publication February 7, 2007; published electronically June 15, 2007. This work was supported by National Science Foundation grant DMS0504691.

http://www.siam.org/journals/sima/39-2/66574.html

${ }^{\dagger}$ Department of Mathematics, University of Pittsburgh, Pittsburgh, PA 15260 (xinfu@pitt.edu).

$\ddagger$ Department of Mathematics, University of Central Florida, Orlando, FL 32816 (yqi@pegasus. cc.ucf.edu).
} 
diffusion for the cubic reaction scheme:

$$
\frac{\partial a}{\partial t}=D_{A} \frac{\partial^{2} a}{\partial x^{2}}-k a b^{2}, \quad \frac{\partial b}{\partial t}=D_{B} \frac{\partial^{2} b}{\partial x^{2}}+k a b^{2},
$$

where $D_{A}$ and $D_{B}$ are the constant diffusion rates of $A$ and $B$, respectively. Initial conditions, in accordance with the observed experiments, are

$$
a(x, 0)=a_{0}, \quad b(x, 0)=g(x) \quad \forall x \in \mathbf{R},
$$

where $a_{0}$ is a positive constant representing the initial uniform distribution of $A$ and $g(x)$ is a nonnegative function with compact support. It is not very difficult to derive from the PDEs that the solution has the following behavior at $x= \pm \infty$ :

$$
a(x, t) \rightarrow a_{0}, \quad b(x, t) \rightarrow 0 \quad \text { as }|x| \rightarrow \infty \quad \forall t \geq 0 .
$$

Introducing dimensionless parameters, dependent and independent variables

$$
D=\frac{D_{B}}{D_{A}}, \quad \bar{a}=\frac{a}{a_{0}}, \quad \bar{b}=\frac{b}{a_{0}}, \quad \bar{t}=k a_{0}^{2} t, \quad \bar{x}=x \sqrt{\frac{k a_{0}^{2}}{D_{A}}}, \quad \bar{g}:=\frac{g}{a_{0}},
$$

and dropping the bars, the initial value problem takes the dimensionless form

$$
\begin{cases}\frac{\partial a}{\partial t}=\frac{\partial^{2} a}{\partial x^{2}}-a b^{2}, & x \in \mathbf{R}, t>0 \\ \frac{\partial b}{\partial t}=D \frac{\partial^{2} b}{\partial x^{2}}+a b^{2}, & x \in \mathbf{R}, t>0 \\ a(x, 0)=1, \quad b(x, 0)=g(x), & x \in \mathbf{R}, t=0 .\end{cases}
$$

Here $D$ measures the rate of diffusion of the autocatalyst relative to that of the reactant.

In the special case $D=1$, the function $a+b$ satisfies a linear heat equation and can be solved explicitly; thus the system is reduced to a single nonlinear equation. For scalar equations, significant results are established and rich theories are available; see, for example, the works of Aronson and Weinberger [2], Chen and Guo [7], Fife and McLeod [8], and Sattinger [18] and the excellent review paper by Xin [23] for detailed information on single equations. The primary concern of the present paper is the case $D \neq 1$, which arises when the chemical species involved have different molecular weights and/or sizes. In particular, enzyme reactions may involve large enzyme molecules and smaller substrate molecules, leading to significantly different rates of diffusion. The system (1.1) also arises in epidemiology (Bailey [3]), where $a$ represents the population density of healthy individuals and $b$ the population density of infected individuals; again, when healthy individuals are significantly more or less mobile than the infected, $D$ is far away from unity.

The wave front propagating phenomenon corresponds to the following behavior of solutions to (1.1): After a certain time of initiation, there are two wave fronts expanding towards $x= \pm \infty$ at a certain speed $v$. In between the two fronts, the reactant is consumed and thus $a \approx 0$; since each unit of reactant consumed produces exactly one unit of autocatalyst, one can expect that $b \approx 1$ inside the wave front. Outside the wave front, the reactant mixture is basically unstirred; thus $a \approx 1$ and $b \approx$ 
0 . Focusing on the right-hand front, one expects that $(a(x, t), b(x, t))=(\alpha(z), \beta(z))$, where $z=x-v t$ and $(\alpha, \beta)$ solves the following system:

$$
\begin{cases}\alpha_{z z}+v \alpha_{z}=\alpha \beta^{2}, \quad \alpha \geq 0 & \forall z \in \mathbf{R}, \\ D \beta_{z z}+v \beta_{z}=-\alpha \beta^{2}, \quad \beta \geq 0 & \forall z \in \mathbf{R}, \\ \lim _{z \rightarrow \infty}(\alpha(z), \beta(z))=(1,0), & \\ \lim _{z \rightarrow-\infty}(\alpha(z), \beta(z))=(0,1) . & \end{cases}
$$

Here $v>0$ is the constant travelling speed.

The Travelling Wave Problem. Given $v>0$, find $(\alpha, \beta) \in\left[C^{2}(\mathbf{R})\right]^{2}$ that satisfies (1.2).

In this paper we study the existence and nonexistence of the travelling waves, which can be generated from the initial value problem (1.1) as just described. One of the most important questions in the study of (1.2) is the existence of a minimum speed travelling wave and the estimate of the minimum speed $v_{\min }$. In particular, for what range of $v$, in relation to $D$, does a travelling wave solution exist?

For quadratic autocatalytic $(A+B \rightarrow 2 B)$, namely, for the travelling wave problem related to the dynamics

$$
\frac{\partial \alpha}{\partial t}=\frac{\partial^{2} \alpha}{\partial x^{2}}-\alpha \beta, \quad \frac{\partial \beta}{\partial t}=D \frac{\partial^{2} \beta}{\partial x^{2}}+\alpha \beta,
$$

Billingham and Needham proved that there is a travelling wave if and only if $v \geq 2 \sqrt{D}$; namely, the minimum wave speed is exactly $2 \sqrt{D}$ (see also the works of Billingham, Merkin, and Needham [4, 5, 14, 15, 16]). Focant and Gallay [9] investigated the existence and stability of travelling waves when both the quadratic and cubic nonlinearities are present in the system.

For the cubic autocatalysis, i.e., for (1.2), the answer is far from complete. Based on an invariant region argument, it was shown in [4] that a travelling wave exists if its speed $v \geq 2 \sqrt{D}$. A more recent work [17] by one of the authors improved the result of [4] to the following: for (1.2),

$$
\begin{aligned}
& \text { (a) there exists a solution if } v \geq\left\{\begin{array}{l}
\sqrt{2 D-1} \text { when } D \geq 1, \\
\sqrt{D} \text { when } D<1 ;
\end{array}\right. \\
& \text { (b) no solution exists if } v \leq\left\{\begin{array}{l}
\sqrt{D / 6} \text { when } D \geq 1, \\
D / \sqrt{6} \text { when } D<1 .
\end{array}\right.
\end{aligned}
$$

This result, which comes out of a much more delicate analysis than that in [4], supplied both upper and lower bounds on minimum wave speed; nevertheless, it is still far from answering the key question of providing a good estimate of minimum speed. In particular, it falls short of providing an accurate order of $v_{\min }$ in terms of small $D$. Numerical simulation by the authors of [4] suggests that $v_{\min } \propto D$ when $D \ll 1$. Furthermore, it is well known that $v_{\min }=1 / \sqrt{2}$ when $D=1$, but neither the results in [4] nor the results in [17] recover this special case from their results of the general case. In this paper we shall provide affirmative answers to these questions and fill in the gap between the general case and the special case of $D=1$.

Theorem 1. Suppose $D<1$. For the travelling wave problem (1.2),

(i) there exists a unique (up to translation) solution if $v \geq \frac{4 D}{\sqrt{1+4 D}}$; 
(ii) there does not exist any solution if $v<\frac{D}{\sqrt{2}}$.

Clearly the above result provides a pretty satisfying bound on the range of wave speeds. In particular, it shows that $v_{\min }(D) \propto D$ for small $D$.

One of the important issues in discussing existence and nonexistence of a travelling wave solution is whether the set of $v$ of the speed for which existence holds is a single interval. While there are heuristic and numerical arguments in [4] demonstrating that the set of admissible wave speed is an interval $\left[v_{\min }, \infty\right)$, for the moment we can only supply a rigorous proof for the case $D \geq 1$.

TheOREm 2. Suppose $D \geq 1$. There exists a positive constant $v_{\min }$ such that (1.2) admits a solution if and only if $v \geq v_{\min }$. In addition, $v_{\min }$ satisfies the estimate

$$
\sqrt{\frac{D}{2}} \leq v_{\min } \leq \sqrt{\frac{D}{1+1 / D}}
$$

It is clear from Theorem 2 that in the special case of $D=1,(1.2)$ admits a solution if and only if $v \geq 1 / \sqrt{2}$.

The general $n$th order isothermal autocatalytic chemical reaction step is governed by the chemical reaction relation

$$
A+n B \rightarrow(n+1) B \quad \text { with rate } k a b^{n} .
$$

We can use the same idea developed in this paper to establish lower bounds for the existence of a travelling wave solution and upper bounds for nonexistence. For this general case, the governing equations are, after proper scaling,

$$
\frac{\partial a}{\partial t}=\frac{\partial^{2} \alpha}{\partial x^{2}}-a b^{n}, \quad \frac{\partial b}{\partial t}=D \frac{\partial^{2} b}{\partial x^{2}}+a b^{n},
$$

where $D=D_{B} / D_{A}$ and the initial value is the same as that in (1.1). The corresponding travelling wave problem is to solve

$$
\begin{cases}\alpha_{z z}+v \alpha_{z}=\alpha \beta^{n}, \quad \alpha \geq 0 & \forall z \in \mathbf{R}, \\ D \beta_{z z}+v \beta_{z}=-\alpha \beta^{n}, \quad \beta \geq 0 & \forall z \in \mathbf{R}, \\ \lim _{z \rightarrow \infty}(\alpha(z), \beta(z))=(1,0), & \\ \lim _{z \rightarrow-\infty}(\alpha(z), \beta(z))=(0,1) . & \end{cases}
$$

Theorem 3. Suppose $D<1$ and $n \geq 2$. A unique (up to translation) travelling wave solution exists for (1.3) if $v \geq 4 D / \sqrt{1+4 D}$. On the other hand, there exists no solution for (1.3) if $v \leq D / \sqrt{K(n)}$, where $K(n)$ is a constant which increases with $n$. In particular, $K(1)=1 / 4, K(2)=2$.

TheOREm 4. Suppose $D \geq 1$ and $n \geq 1$. There exists a positive constant $v_{\min }$ such that (1.3) admits a travelling wave if and only if $v \geq v_{\min }$. In addition, $v_{\min }$ is bounded by

$$
\sqrt{\frac{D}{K(n)}} \leq v_{\min } \leq \sqrt{\frac{D}{K(n)}} \frac{1}{\sqrt{1-\left(1-\frac{1}{D}\right) \frac{\sqrt{4 K(n)+1}-1}{\sqrt{4 K(n)+1}+1}}},
$$

where $K(n)$ is the same constant as in Theorem 3. 
We note in passing the recent works to study the spatiotemporal profiles of $L^{1}$ initial values by Bricmont, Kupiainen, and Xin [6] and the steady-state solutions by Shi and Wang [21].

The organization of this paper is as follows. Section 2 contains preliminary analysis and an outline of our approach. The case $D \geq 1$ is discussed in section 3 , and the case $D<1$ in section 4 .

\section{Preliminary.}

2.1. A scalar equation. First we review the existence of a travelling wave solution of unit speed to the equation

$$
u_{z z}+u_{z}=k u(1-u)^{n}, \quad 0 \leq u \leq 1 \quad \text { on } \mathbf{R}, \quad u(-\infty)=0, \quad u(\infty)=1 .
$$

Here $n \geq 1$ is a parameter, and $k$ is a positive constant. We seek upper bounds on $k$ for the existence of a solution. Since a solution, if it exists, satisfies $u_{z}>0$ on $\mathbf{R}$, we can write $u^{\prime}=Q(u)$ and work on the $(u, Q)$ phase plane. The resulting equation on the phase plane is

$$
\left\{\begin{array}{l}
Q Q^{\prime}+Q=k u(1-u)^{n} \quad \forall u \in[0,1], \\
Q(0)=0, \quad Q>0 \text { on }(0,1) .
\end{array}\right.
$$

There is a one-to-one correspondence between solutions to (2.1) and solutions to (2.2) satisfying the additional requirement $Q(1)=0$.

Lemma 2.1. For each $n \geq 1$ and $k>0$, there exists a unique solution $Q=$ $Q(n, k ; \cdot)$ to $(2.2)$. In addition, there exists a positive constant $K(n)$ such that $Q(n, k ; 1)=0$ if $k \in(0, K(n)]$ and $Q(n, K ; 1)>0$ if $k \in(K(n), \infty)$. Consequently, (2.2) admits a solution if and only if $k \in(0, K(n)]$.

In addition, $K(n)$ is a strictly increasing function of $n$ and $K(1)=\frac{1}{4}, K(2)=2$.

Proof. The existence of $Q$ and $K$ follows by the comparison principle. The exact value of $K(1)$ is calculated by a known fact that the function $K(1) u(1-u)$ is concave, and thus the minimum wave speed $v=1$ satisfies $1=2 \sqrt{K(1)}$; hence $K(1)=1 / 4$. In the case $n=2$, the exact solution is given by $Q=u(1-u)$; thus $K(2)=2$. We omit details, because it is a standard argument.

2.2. Basic properties of travelling waves. Suppose $(v, \alpha, \beta)$ solves (1.3). Then $\left[\alpha_{z}+v \alpha+D \beta_{z}+v \beta\right]_{z}=0$, so that $\alpha_{z}+D \beta_{z}+v(\alpha+\beta)$ is a constant function. Using the boundary conditions, we find that

$$
\alpha_{z}+D \beta_{z}+v(\alpha+\beta-1)=0 \quad \text { on } \mathbf{R} .
$$

With the new variable $w=\beta_{z}$, (1.3) is equivalent to the following third order ODE system

$$
\left\{\begin{array}{l}
\alpha_{z}=v(1-\alpha-\beta)-D w \\
\beta_{z}=w \\
w_{z}=-D^{-1}\left(\alpha \beta^{n}+v w\right) \\
\lim _{z \rightarrow \infty}(\alpha(z), \beta(z), w(z))=(1,0,0) \\
\lim _{z \rightarrow-\infty}(\alpha(z), \beta(z), w(z))=(0,1,0) .
\end{array}\right.
$$

It is clear that in the $(\alpha, \beta, w)$ phase space, there are two equilibrium points: $(0,1,0)$ and $(1,0,0)$. The following are a few basic properties of travelling wave solutions. 
Proposition 1. The systems (1.3) and (2.3) are equivalent. Any solution $(\alpha, \beta)$ to (1.3) or $(\alpha, \beta, w)$ to (2.3) has the following properties:

(1) $\alpha_{z}>0>\beta_{z}$ on $\mathbf{R}$.

(2) $\alpha+\beta<1$ on $\mathbf{R}$ if $D<1, \alpha+\beta \equiv 1$ if $D=1$, and $\alpha+\beta>0$ if $D>1$.

(3) $v=\int_{-\infty}^{\infty} \alpha(z) \beta^{n}(z) d z>0$.

(4) The equilibrium point $(0,1,0)$ of $(2.3)$ is a saddle with a two-dimensional stable manifold and a one-dimensional unstable manifold. The eigenvalues and associated eigenvectors are

$$
\begin{array}{ll}
\lambda_{1}=-v D^{-1}, & \mathbf{e}_{\lambda_{1}}=\left(0,-1,-\lambda_{1}\right)^{T}, \\
\lambda_{2}=-\frac{1}{2}\left(\sqrt{v^{2}+4}+v\right), & \mathbf{e}_{\lambda_{2}}=\left(\lambda_{2}\left(D \lambda_{2}+v\right),-1,-\lambda_{2}\right)^{T}, \\
\lambda_{3}=\frac{1}{2}\left(\sqrt{v^{2}+4}-v\right), & \mathbf{e}_{\lambda_{3}}=\left(\lambda_{3}\left(D \lambda_{3}+v\right),-1,-\lambda_{3}\right)^{T} .
\end{array}
$$

(5) When $n>1$, the equilibrium point $(1,0,0)$ is degenerate; it has a twodimensional stable manifold and a one-dimensional center manifold. The eigenvalues and associated eigenvectors are

$$
\begin{array}{ll}
\mu_{1}=-v, & \mathbf{e}_{\nu_{1}}=(1,0,0)^{T}, \\
\mu_{2}=-v D^{-1}, & \mathbf{e}_{\nu_{2}}=\left(0,-1,-v D^{-1}\right)^{T}, \\
\mu_{3}=0, & \mathbf{e}_{\nu_{3}}=(1,-1,0)^{T} .
\end{array}
$$

All items except (3) were proved in [4]. The equation in property (3) is obtained by integrating the equation involving $\alpha_{z z}$ in (1.3) with the boundary conditions $\alpha(\infty)=1$ and $\alpha(-\infty)=0$.

The third property in the proposition demonstrates that $v>0$. The fourth property clearly tells us that the travelling wave we are looking for is indeed the onedimensional unstable manifold associated with the equilibrium $(0,1,0)$. Hence, given $v>0$, a travelling wave of speed $v$, if it exists, is unique up to a translation.

2.3. New setting - A nonautonomous $2 \times 2$ system. Unlike in earlier works $[4,17]$, here we shall use a transformation to turn the third order autonomous system (2.3) into a second order nonautonomous system, using $u:=1-\beta$ as the independent variable. This is allowed since for the solution of interest, $\beta_{z}<0$, and thus $z \rightarrow 1-\beta(z)$ has an inverse. To make the resulting system as simple as possible, we also scale the other variables. Hence, we introduce

$$
u=1-\beta, \quad A=\frac{D \alpha}{v^{2}}, \quad y=\frac{v z}{D}, \quad \kappa:=\frac{D}{v} .
$$

The system of differential equations (1.3) becomes

$$
\begin{cases}u_{y y}+u_{y}=A(1-u)^{n} & \text { on } \mathbf{R}, \\ A_{y}=\kappa^{2}\left(u+u_{y}\right)-D A & \text { on } \mathbf{R} .\end{cases}
$$

Since $u_{y}>0$ for the solution of interest, we can use $u$ as the independent variable. Introducing $P(u)=u_{y}$, we have an equivalent system of second order nonautonomous (singular) ODEs:

$$
\begin{cases}P P^{\prime}=A[1-u]^{n}-P & \forall u \in[0,1], \\ P A^{\prime}=\kappa^{2}[P+u]-D A & \forall u \in[0,1], \\ P(u)>0, A(u)>0 & \forall u \in(0,1), \\ P(0)=0, A(0)=0 . & \end{cases}
$$


Lemma 2.2. For every $D>0$ and $\kappa>0$, (2.4) admits a unique solution. In addition,

$$
P(u)=\lambda u+O\left(u^{2}\right), \quad A(u)=\lambda(1+\lambda) u+O\left(u^{2}\right) \quad \text { as } u \searrow 0,
$$

where

$$
\lambda:=\frac{1}{2}\left(\sqrt{4 \kappa^{2}+D^{2}}-D\right) \quad\left(\text { the only positive root to } \lambda(\lambda+D)=\kappa^{2}\right) .
$$

Furthermore, $A^{\prime}(u)>0$ for all $u \in[0,1)$, and there are only two possible cases:

(a) $P(1)>0$ : there does not exist any travelling wave solution to (1.3).

(b) $P(1)=0$ : there exists a travelling wave solution to (1.3), unique up to translation.

Proof. We divide the proof into several steps.

1. A solution to (2.4) corresponds exactly to the part of the one-dimensional unstable manifold associated with the equilibrium point $(0,1,0)$ of the autonomous system (2.3) that has the property $\alpha, \beta>0$ and $w<0$. Hence, for some $\delta \in(0,1]$, $(2.4)$ admits a unique solution in $[0, \delta)$. The solution satisfies the asymptotic expansion (2.5) and can be extended as long as $P>0$.

2. It is easy to see that $A$ cannot hit zero before $P$ does, since otherwise, $P A^{\prime}=$ $\kappa^{2}(p+u)>0$ at $A=0$ and $P>0$, which is impossible. If $\lim _{u>\delta} P(u)=0$ at some $\delta \in(0,1)$, then $\liminf _{u \succ \delta} P(u) P^{\prime}(u) \leq 0$, and thus $A(\delta):=\lim _{u \succ \delta} A(u)=0$. But the equation for $A$ gives $P A^{\prime}>\kappa^{2} \delta / 2>0$ for all $u$ sufficiently close to $\delta$ from below, which contradicts $A(\delta)=0$. Since the system has at most a linear growth in $P$ and $A$, the solution can be uniquely extended to $[0,1)$ and $P>0, A>0$ in $(0,1)$.

3. Now we show that $A^{\prime}>0$ on $[0,1)$. From the asymptotic behavior $(2.5)$, $A \in C^{1}([0,1))$ and $A^{\prime}(0)=\lambda(\lambda+1)>0$. Also a combination of the two equations in (2.4) yields

$$
P\left[\kappa^{2}(P+u)-D A\right]^{\prime}=-D\left[\kappa^{2}(P+u)-D A\right]+\kappa^{2} A(1-u)^{n} .
$$

Gronwall's inequality then gives $\kappa^{2}(P+u)-D A>0$ in $(0,1)$. Thus,

$$
0<A<D^{-1} \kappa^{2}(P+u) \text { in }(0,1), \quad A^{\prime}>0 \text { in }[0,1) .
$$

4. Since $(P+u)^{\prime}=A(1-u)^{n} / P>0$ in $(0,1), P+u$ is strictly increasing in $(0,1)$ so that $\lim _{u \nearrow 1} P(u)$ exists. To show that it is finite, observe that when $P \geq 1$,

$$
[P+u]^{\prime} \leq P[P+u]^{\prime}=A(1-u)^{n} \leq D^{-1} \kappa^{2}(P+u)(1-u)^{n} .
$$

This implies that $P+u$ is bounded uniformly in $u \in[0,1)$; thus $P(1):=\lim _{u \nearrow 1} P(u)$ exists and is finite. Consequently, $A(1):=\lim _{u / 1} A(u)$ also exists and is finite.

5. If $P(1)>0$, we have a classical solution of $(2.4)$ on $[0,1]$. Since a travelling wave is required to have $u=1-\beta \leq 1$, we see that there is no travelling wave solution to (1.3).

6. Suppose $P(1)=0$. Since $[P+u]^{\prime}>0$ in $(0,1)$, we have $P(u)+u<P(1)+1=1$; i.e., $P(u)<1-u$ for all $u \in[0,1)$. Since $\kappa^{2}(P+u)-D A>0$ in $(0,1)$, we see that

$$
\begin{aligned}
A(1) & =\int_{0}^{1} \frac{\kappa^{2}[P(u)+u]-D A(u)}{P(u)} d u \\
& \geq \int_{0}^{1} \frac{\kappa^{2}[P(u)+u]-D A(u)}{1-u} d u \geq \int_{0}^{1} \frac{\kappa^{2}[u-1]}{1-u} d u+\int_{0}^{1} \frac{\kappa^{2}-D A(u)}{1-u} d u .
\end{aligned}
$$


Since $D A(u)<D A(1) \leq \kappa^{2}(P(1)+1)=\kappa^{2}$ for all $u \in(0,1)$, for the last integral to be convergent, we must have $A(1)=\kappa^{2} / D$. It is then easy to see that $\alpha=D A / \kappa^{2} \rightarrow 1$ as $u \rightarrow 1$. Transferring back to the original variable $z$, we then obtain a travelling wave solution to (1.3).

In what follows, we shall estimate upper and lower bounds of $A / u$; thus Lemma 2.1 can be applied to generate upper and lower bounds of $v_{\min }$.

3. The case $D \geq 1$. In this section we deal with the case of $D \geq 1$.

Lemma 3.1. Suppose $D \geq 1$. Then $D A(u) \geq \kappa^{2} u$ for all $u \in[0,1]$. Consequently, there is no travelling wave solution to (1.3) when $\kappa^{2}>D K(n)$, i.e., when $v<\sqrt{D / K(n)}$.

Proof. If $D=1, A(u)=\kappa^{2} u$ for all $u \in[0,1]$. When $D>1$, for every $u \in(0,1)$,

$$
P\left[D A-\kappa^{2} u\right]^{\prime}=-D\left[D A-\kappa^{2} u\right]+(D-1) \kappa^{2} P>-D\left[D A-\kappa^{2} u\right] .
$$

In addition, when $u$ is sufficiently small, $D A(u)=D(1+\lambda) \lambda u+O\left(u^{2}\right)>[D+\lambda] \lambda u=$ $\kappa^{2} u$. Applying Gronwall's inequality, we derive that $D A>\kappa^{2} u$ on $(0,1)$.

Now suppose $\kappa^{2}>D K(n)$. Let $\hat{k} \in\left(K(n), k^{2} / D\right)$. Then $A(u) \geq \hat{k} u$ on $[0,1]$ so that

$$
P P^{\prime}+P=A(1-u)^{n} \geq \hat{k} u(1-u)^{n} \quad \forall u \in[0,1] .
$$

We compare $P(u)$ and the solution $Q(n, \hat{k} ; u)$ given in Lemma 2.1. Using a Taylor expansion, we can show that $P(u)>Q(n, \hat{k} ; u)$ for all $u \in(0, \epsilon]$ for some $\epsilon>0$. In the interval $[\epsilon, 1]$ we can use the regular comparison principle to show that $P(u)>$ $Q(n, \hat{k} ; u)$ for all $u \in[\epsilon, 1)$. In particular, $P(1) \geq Q(n, \hat{k} ; 1)>0$, so that there is no travelling wave solution to (1.3). Since $\kappa=D / v$, the condition $\kappa^{2}>D K(n)$ is the same as $v<\sqrt{D / K(n)}$.

Lemma 3.2. Suppose $D>1$. Then,

$$
A(u)<\lambda(1+\lambda) u, \quad P(u)<\lambda u \quad \forall u \in(0,1) .
$$

Consequently, there exists a travelling wave solution to (1.3) when $\lambda(\lambda+1) \leq K(n)$, i.e., when

$$
v \geq \sqrt{\frac{D}{K(n)}} \frac{1}{\sqrt{1-\left(1-\frac{1}{D}\right) \frac{\sqrt{4 K(n)+1}-1}{\sqrt{4 K(n)+1}+1}}} .
$$

Proof. A higher order Taylor expansion near $u=0$ shows that $A<\lambda(\lambda+1) u$ and $P<\lambda u$ for all sufficient small positive $u$. Set

$$
\hat{B}=\sup \{b \in(0,1) \mid P(u)<\lambda u, \quad A(u)<\lambda(1+\lambda) u \quad \forall u \in(0, b)\} .
$$

We show that $\hat{B}=1$. Suppose on the contrary that $\hat{B}<1$. Then either $P(\hat{B})-\lambda \hat{B}=0$ or $A(\hat{B})-\lambda(1+\lambda) \hat{B}=0$. In $(0, \hat{B}]$,

$$
\begin{aligned}
P[A-\lambda(1+\lambda) u]^{\prime} & =\kappa^{2}(P+u)-D A-\lambda(1+\lambda) P \\
& =\lambda(D+\lambda)(P+u)-D A-\lambda(1+\lambda) P \\
& =-D[A-\lambda(1+\lambda) u]+\lambda(D-1)(P-\lambda u) \\
& \leq-D[A-\lambda(1+\lambda) u] .
\end{aligned}
$$


Gronwall's inequality then implies that $A<\lambda(\lambda+1) u$ on $(0, \hat{B}]$. Similarly, for all $u \in(0, \hat{B}]$,

$$
\begin{aligned}
P[P-\lambda u]^{\prime} & =-(1+\lambda) P+A(1-u)^{n} \\
& =-(1+\lambda)(P-\lambda u)-\lambda(1+\lambda) u+A(1-u)^{n} \\
& <-(1+\lambda)(P-\lambda u) .
\end{aligned}
$$

Gronwall's inequality shows that $P<\lambda u$ on $(0, \hat{B}]$. We reach a contradiction. This proves that $\hat{B}=1$; i.e., $P(u)<\lambda u$ and $A(u)<\lambda(1+\lambda) u$ for all $u \in(0,1)$.

Suppose $\lambda(1+\lambda) \leq K(n)$. We can use comparison to show that $P(u) \leq Q(n, K(n)$; $u$ ) for all $u \in[0,1]$ so that $P(1)=0$. Namely, there exists a travelling wave solution to $(1.3)$. $\quad \square$

Proof of Theorem 2. This is a special case of Theorem 4, by setting $n=2$ and acknowledging that $K(2)=2$.

Proof of Theorem 4 . The estimate of $v_{\min }$, when it exists, follows from the above two lemmas.

We notice that the set of admissible speed is a closed set. Indeed, if there is no travelling wave of speed $\hat{v}>0$, then the solution $(P, A)$ to $(2.4)$ with $v=\hat{v}$ has the property that $P(1)>0$. It then follows by continuous dependence that for any $v$ sufficiently close to $\hat{v}$, the solution to (2.4) also satisfies $P(1)>0$. This implies that there is no travelling wave of speed $v$ for any $v$ sufficiently close to $\hat{v}$. Thus the complement of the set of admissible speed is open; that is, the set of admissible speed is closed.

Hence, to show the existence of $v_{\min }$, it suffices to show that if $v_{1}>v_{0}$ and there exists a travelling wave of speed $v_{0}$, then there also exists a travelling wave of speed $v_{1}$. For this, we denote $\kappa_{i}=D / v_{i}$ and $\left(P_{i}, A_{i}\right)$ the solution to (2.4) with $\kappa=D / v_{i}$, $i=0,1$. The existence of a travelling wave of speed $v_{0}$ implies that $P_{0}(1)=0$. To show that there exists a travelling wave of speed $v_{1}$, it is necessary and sufficient to show that $P_{1}(1)=0$. For this, it suffices to show that $P_{1}<P_{0}$ in $(0,1)$.

Notice that $\kappa_{1}<\kappa_{0}$. Denote by $\lambda_{i}$ the positive root to $\lambda_{i}\left(\lambda_{i}+D\right)=\kappa_{i}^{2}$. Then $\lambda_{1}<\lambda_{0}$. The asymptotic expansion for $(P, A)$ then implies that there exists $\epsilon>0$ such that for $u \in(0, \epsilon], P_{1}(u)<P_{0}(u)$ and $A_{1}(u)<A_{0}(u)$. In addition, for small $u$, the functions $\alpha_{i}:=D A_{i} / \kappa_{i}^{2}$ satisfy

$$
\begin{aligned}
\alpha_{0}-\alpha_{1} & =\left\{\frac{D \lambda_{0}\left(\lambda_{0}+1\right)}{\kappa_{0}^{2}}-\frac{D \lambda_{1}\left(\lambda_{1}+1\right)}{\kappa_{1}^{2}}\right\} u+O\left(u^{2}\right) \\
& =D\left\{\frac{\lambda_{0}+1}{\lambda_{0}+D}-\frac{\lambda_{1}+1}{\lambda_{1}+D}\right\} u+O\left(u^{2}\right) \\
; 6 p t] & =\frac{D(D-1)\left(\lambda_{0}-\lambda_{1}\right)}{\left(\lambda_{0}+D\right)\left(\lambda_{1}+D\right)} u+O\left(u^{2}\right)>0
\end{aligned}
$$

since $D>1$ and $\lambda_{0}>\lambda_{1}$. Now let

$$
\hat{B}=\sup \left\{b \in(0,1) \mid P_{1}(u)<P_{0}(u) \forall u \in(0, b)\right\} .
$$

We claim that $\hat{B}=1$. Suppose the contrary, $\hat{B}<1$. Then $P_{0}(\hat{B})=P_{1}(\hat{B})>0$.

First we claim that $A_{0}>A_{1}$ on $(0, \hat{B}]$. Suppose it is not true; then there is a $u_{1} \in(0, \hat{B}]$ at which $A_{0}\left(u_{1}\right)=A_{1}\left(u_{1}\right)$. Since $\kappa_{0}>k_{1}$, there exists $u_{2} \in\left(0, B_{1}\right)$ such that $\alpha_{0}\left(u_{2}\right)=\alpha_{1}\left(u_{2}\right)$ and $\alpha_{0}\left(u_{2}\right)^{\prime} \leq \alpha_{1}\left(u_{2}\right)^{\prime}$. But, at $u=u_{2}$,

$$
\left[\alpha_{0}-\alpha_{1}\right]^{\prime}=\frac{D\left(u-\alpha_{0}\right)}{P_{0}}-\frac{D\left(u-\alpha_{1}\right)}{P_{1}}=\frac{D\left(\alpha_{0}-u\right)\left(P_{0}-P_{1}\right)}{P_{0} P_{1}}>0
$$


since $\alpha_{0}-u=\left[D A_{0}-\kappa_{0}^{2} u\right] / \kappa_{0}^{2}>0$ by Lemma 3.1 and $P_{0}>P_{1}$ in $(0, \hat{B}) \ni u_{2}$. Thus, we must have $A_{0}>A_{1}$ in $[0, \hat{B}]$. Consequently, we obtain from the equation for $P_{i}$ that

$$
\frac{1}{2}\left[P_{1}^{2}-P_{0}^{2}\right]^{\prime}=\left[P_{0}-P_{1}\right]+\left(A_{1}-A_{0}\right)[1-u]^{n}<\left[P_{0}-P_{1}\right]=\frac{P_{0}^{2}-P_{1}^{2}}{P_{0}+P_{1}} .
$$

Gronwall's inequality on $[\epsilon, \hat{B}]$ then gives $P_{1}^{2}-P_{0}^{2}<0$ on $[\epsilon, \hat{B}]$, contradicting $P_{0}(\hat{B})=$ $P_{1}(\hat{B})$. Hence, $\hat{B}=1$ and $P_{1}<P_{0}$ on $(0,1)$. This completes the proof of Theorem 4 . 口

4. The case of $D<1$. In this section, we establish the results on the case of $D<1$.

Lemma 4.1. Suppose $D<1$. Then $A>\kappa^{2} u$ on $(0,1)$. Consequently, when $\kappa^{2}>K(n)$, i.e., $v<D / \sqrt{K(n)}$, there is no travelling wave solution to (1.3).

Proof. Direct calculation shows that

$$
\begin{aligned}
P\left[A-\kappa^{2} u\right]^{\prime} & =\kappa^{2}(P+u)-D A-\kappa^{2} P=\kappa^{2}(1-D) u-D\left(A-\kappa^{2} u\right) \\
& >-D\left(A-\kappa^{2} u\right) \quad \forall u \in(0,1) .
\end{aligned}
$$

Since $A=\lambda(1+\lambda) u+O\left(u^{2}\right)>\kappa^{2} u$, for all sufficiently small positive $u$, Gronwall's inequality gives $A>\kappa^{2} u$ on $[0,1)$.

One can show that $P(u)>Q\left(n, k^{2} ; u\right)$ for all $u \in(0,1)$ by first using an asymptotic expansion at $u=0$ for $0<u \leq \epsilon$ and then a comparison principle for the differential equation in $(\epsilon, 1)$.

It then follows from Lemma 2.1 that when $\kappa^{2}>K(n)$, we must have $P(1) \geq Q\left(n, k^{2} ; 1\right)>0$; i.e., there does not exist any solution to the travelling wave problem.

To establish the existence of a solution, we need to find an upper bound of $A$. Although there is the estimate $A<\kappa^{2}(u+P) / D$ available for use, we are not satisfied with such an estimate since when $D$ is very small, it is not sufficient to show that $v_{\min }=O(D)$. Hence, we seek another bound.

Lemma 4.2. Suppose $D<1$. Then $A(u)(1-u)^{n / 2} \leq \lambda[P(u)+u] \forall u \in[0,1)$.

Proof. When $u=0$, the two sides are equal. Computation shows that, in $(0,1]$,

$$
\begin{aligned}
& P\left[(1-u)^{n / 2} A-\lambda(P+u)\right]^{\prime} \\
= & (1-u)^{n / 2}\left[\kappa^{2}(P+u)-D A\right]-\frac{1}{2} n P A(1-u)^{n / 2-1}-\lambda A(1-u)^{n} \\
\leq & -\left[D+\lambda(1-u)^{n / 2}\right]\left[A(1-u)^{n / 2}-\lambda(P+u)\right]+(P+u)\left[\left(\kappa^{2}-\lambda^{2}\right)(1-u)^{n / 2}-\lambda D\right] \\
= & -\left[D+\lambda(1-u)^{n / 2}\right]\left[A(1-u)^{n / 2}-\lambda(P+u)\right]-\lambda D(P+u)\left[1-(1-u)^{n / 2}\right] \\
\leq & -\left[D+\lambda(1-u)^{n / 2}\right]\left[A(1-u)^{n / 2}-\lambda(P+u)\right] .
\end{aligned}
$$

Here we have dropped the term $\frac{1}{2} n P(1-u)^{n / 2-1}$ in the first inequality and used $\kappa^{2}=\lambda(\lambda+D)$ in the second inequality. The assertion of the lemma thus follows from Gronwall's inequality.

Proof of Theorem 3. The nonexistence follows directly from Lemma 4.1. We now prove the existence. Simple computation shows that $v \leq 4 D / \sqrt{1+4 D}$ is equivalent to $\lambda \leq 1 / 4$. We proceed to show that $P-u(1-u) / 2 \leq 0$ on $(0,1)$. It is easy to verify, 
using the result of Lemma 4.2, that

$$
\begin{aligned}
P[2 P-u(1-u)]^{\prime}= & P(2 u-3)+2 A(1-u)^{n} \\
\leq & P(2 u-3)+2 \lambda(P+u)(1-u)^{n / 2} \\
= & {\left[u-3 / 2+\lambda(1-u)^{n / 2}\right][2 P-u(1-u)] } \\
& \quad+u(1-u)\left[2 \lambda(1-u)^{n / 2-1}+\lambda(1-u)^{n / 2}+u-3 / 2\right] \\
& <\left[u-3 / 2+\lambda(1-u)^{n / 2}\right][2 P-u(1-u)],
\end{aligned}
$$

since $\lambda \leq 1 / 4$ and $n \geq 2$ yield

$$
\begin{aligned}
& 2 \lambda(1-u)^{n / 2-1}+\lambda(1-u)^{n / 2}+u-3 / 2 \\
\leq & 2 \lambda+\lambda(1-u)+u-3 / 2 \\
= & 2 \lambda-1 / 2+(\lambda-1)(1-u) \leq 0 .
\end{aligned}
$$

Because $2 P<u(1+u)$ for small $u$, Gronwall's inequality shows that $P<u(1-u) / 2$ on $(0,1)$. Thus $P(1)=0$. This proves the existence and completes the proof of the theorem.

Finally, Theorem 1 is a special case of Theorem 3 with $n=2$.

Acknowledgments. The authors thank J. B. McLeod and Yi Li for stimulating discussions.

\section{REFERENCES}

[1] R. Aris, P. Gray, And S. K. Scott, Modelling of cubic autocatalysis by successive biomolecular steps, Chem. Eng. Sci., 43 (1988), pp. 207-211.

[2] D. G. Aronson And H. F. Weinberger, Multidimensional diffusion arising in population genetics, Adv. Math., 30 (1978), pp. 33-76.

[3] N. J. T. BAILEY, The Mathematical Theory of Infectious Diseases and its Applications, Griffin, London, 1975.

[4] J. Billingham AND D. J. NeEdham, The development of travelling waves in quadratic and cubic autocatalysis with unequal diffusion rates. I. Permanent form travelling waves, Philos. Trans. Roy. Soc. London Ser. A, 334 (1991), pp. 1-24.

[5] J. Billingham And D. J. Needham, The development of travelling waves in quadratic and cubic autocatalysis with unequal diffusion rates. II. An initial value problem with an immobilized or nearly immobilized autocatalyst, Philos. Trans. Roy. Soc. London Ser. A, 336 (1991), pp. 497-539.

[6] J. Bricmont, A. Kupiainen, And J. Xin, Global large time self-similarity of a thermal-diffusive combustion system with critical nonlinearity, J. Differential Equations, 130 (1996), pp. 935 .

[7] X. Chen And J.-S. Guo, Existence and asymptotic stability of traveling waves of discrete quasilinear monostable equations, J. Differential Equations, 184 (2002), pp. 549-569.

[8] P. C. Fife AND J. B. MCLEOD, The approach of nonlinear diffusion equations to travelling wave front solutions, Arch. Rational Mech. Anal., 65 (1977), pp. 335-361.

[9] S. Focant And Th. Gallay, Existence and stability of propagating fronts for an autocatalytic reaction-diffusion system, Phys. D, 120 (1998), pp. 346-368.

[10] R. J. Gowland and G. Stedman, A novel moving boundary reaction involving hydoxylamine and nitric acid, J. Chem. Soc. Chem. Commun., 10 (1983), pp. 1038-1039.

[11] P. Gray, J. F. Griffiths, and S. K. Scott, Experimental studies of the ignition diagram and the effect of added hydrogen, Proc. R. Soc. Lond. Ser. A, 397 (1984), pp. 21-44.

[12] P. Gray and S. K. Scott, Chemical Oscillations and Instabilities, Clarendon Press, Oxford University Press, Oxford, UK, 1990.

[13] A. Hanna, A. Saul, And K. Showalter, Detailed studies of propagating fronts in the iodate oxidation of arsenous acid, J. Am. Chem. Soc., 104 (1982), pp. 3838-3844.

[14] J. H. Merkin And D. J. Needham, Propagating reaction-diffusion waves in a simple isothermal quadratic autocatalytic chemical system, J. Engrg. Math., 23 (1989), pp. 343-356. 
[15] J. H. Merkin And D. J. NeEdham, The development of travelling waves in a simple isothermal chemical system. II. Cubic autocatalysis with quadratic and linear decay, Proc. Roy. Soc. London Ser. A, 430 (1990), pp. 315-345.

[16] J. H. Merkin AND D. J. NeEdham, The development of travelling waves in a simple isothermal chemical system. IV. Cubic autocatalysis with quadratic decay, Proc. Roy. Soc. London Ser. A, 434 (1991), pp. 531-554.

[17] Y. W. QI, The development of travelling waves in cubic auto-catalysis with different rates of diffusion, Phys. D, 226 (2007), pp. 129-135.

[18] D. Sattinger, On the stability of waves of nonlinear parabolic systems, Adv. Math., 22 (1976), pp. 312-355.

[19] A. Saul and K. Showalter, Propagating reaction-diffusion fronts, in Oscillations and Travelling Waves in Chemical Systems, R. J. Field and M. Burgern, eds., Wiley, New York, 1984.

[20] V. G. E. E. SeL'Kov, Euro. J. Biochem., 4 (1968), p. 79.

[21] J. SHI AND X. WANG, Hair-triggered instability of radial steady states, spread and extinction in semilinear heat equations, J. Differential Equations, 231 (2006), pp. 235-251.

[22] V. G. Voronkov and N. N. Semenov, Zh. Fiz. Khim., 13 (1939), p. 1695.

[23] J. XIN, Front propagation in heterogeneous media, SIAM Rev., 42 (2000), pp 161-230.

[24] A. N. Zaikin AND A. M. Zhabotinskir, Concentration wave propagation in two-dimensional liquid-phase self-oscillating system, Nature, 225 (1970), pp. 535-537. 